\title{
Identification of key genes and pathways in regulating immune-induced diseases of dendritic cells by bioinformatic analysis
}

\author{
YANG ZHENG $^{1,2^{*}}$, XIANGHUI ZHENG ${ }^{1,2^{*}}$, SHUANG LI ${ }^{1,2}$, HANLU ZHANG $^{1,2}$, MINGYANG LIU $^{1,2}$,

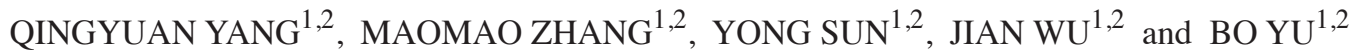 \\ ${ }^{1}$ Department of Cardiology, The Second Affiliated Hospital of Harbin Medical University; ${ }^{2}$ The Key Laboratory of \\ Myocardial Ischemia, Harbin Medical University, Ministry of Education, Harbin, Heilongjiang 150001, P.R. China
}

Received December 19, 2017; Accepted March 22, 2018

DOI: $10.3892 / \mathrm{mmr} .2018 .8834$

\begin{abstract}
Dendritic cells (DCs) serve crucial roles in the activation of the immune response, and imbalance in the activation or inhibition of DCs has been associated with an increased susceptibility to develop immune-induced diseases. However, the molecular mechanisms of regulating immune-induced diseases of DCs are not well understood. The aim of the present study was to identify the gene signatures and uncover the potential regulatory mechanisms in DCs. A total of 4 gene expression profiles (GSE52894, GSE72893, GSE75938 and GSE77969) were integrated and analyzed in depth. In total, 241 upregulated genes and 365 downregulated genes were detected. Gene ontology and pathway enrichment analysis showed that the differentially expressed genes (DEGs) were significantly enriched in the inflammatory response, the
\end{abstract}

Correspondence to: Dr Jian Wu, Department of Cardiology, The Second Affiliated Hospital of Harbin Medical University, 246 Xuefu Road, Nangang, Harbin, Heilongjiang 150001, P.R. China

E-mail:wujian780805@163.com

*Contributed equally

Abbreviations: DCs, dendritic cells; mDCs, mature DCs; imDCs, immature DCs; DEGs, differentially expressed genes; GVHD, graft-versus-host disease; APCs, antigen presenting cells; AS, atherosclerosis; MHC, major histocompatibility complex; PAMPs, pathogen-associated molecular patterns; DAMPs, damage-associated molecular patterns; PPRs, pattern recognition receptors; TLRs, toll-like receptors; Treg, regulatory T; NPC, nasopharyngeal carcinoma; GEO, gene expression omnibus; GO, gene ontology; PPI, protein-protein interaction; KEGG, Kyoto Encyclopedia of Genes and Genomes; STRING, Search Tool for the Retrieval of Interacting Genes; MCODE, Molecular Complex Detection; PBMCs, peripheral blood mononuclear cells; Mo-DCs, monocyte-derived dendritic cells; LPS, lipopolysaccharides; miRNAs, microRNAs

Key words: dendritic cell, immune-induced diseases, differentially expressed genes, protein-protein network, hub genes tumor necrosis factor (TNF) signaling pathway, the nuclear factor $(\mathrm{NF})-\kappa \mathrm{B}$ signaling pathway and antigen processing. The top 10 hub genes were identified from the protein-protein analysis. The most significant 2 modules were filtered from the protein-protein network. The genes in 2 modules were involved in type I interferon signaling, the $\mathrm{NF}-\kappa \mathrm{B}$ signaling pathway and the TNF signaling pathway. Furthermore, the microRNA-mRNA network analysis was performed. The results of the present study revealed that the identified DEGs and pathways may improve our understanding of the mechanisms of the maturation of DCs, and the candidate hub genes that may be therapeutic targets for immune-induced diseases.

\section{Introduction}

The immune-mediated diseases including pathogens, atherosclerosis, autoimmunity, sel-tolerance, graft-versus-host disease (GVHD) and cancer have increased with socio-economic. As professional antigen presenting cells (APCs), Dendritic cells (DCs) are central regulators of innate and adaptive immune responses. Since their discovery by Steinman RM in 1973 (1), DCs have been demonstrated as key components in immune response directed against cancer cells, pathogens, allergens and autoantigens (2). During the past decades, increasingly evidences have certified that DCs activate the immune responses and result in immune-related diseases. Atherosclerosis (AS) is a chronic inflammatory disease of vessel wall. DCs can be observed in arterial vessels of AS. Some studies have demonstrated that DCs can take up lipids $(3,4)$, control cholesterol homeostasis (5) and enhance T-cell activation in AS (6). Our previous studies have addressed the role of DCs in GVHD. MicroRNA let-7i-5p was upregulated in mature DCs (mDCs), and let-7i-5p-inhibitor depressed the maturation of DCs (7). In another study, we have demonstrated that let-7i-5p regulated DCs maturation through targeting $I L-10$ via the JAK1-STAT3 signal pathway (8). Moreover, transfusion let-7i-5p-inhibitor DCs can prolong cardiac allograft survival in a rat heart transplantation model (8).

DCs have two different functions depending on their maturation status. MDCs with high levels of major 
histocompatibility complex (MHC) and costimulatory molecules have been identified as potent immune activators of Ag-specific immune response, while, immature DCs (imDCs) expressed low expression of MHC and costimulatory molecules that maintain immune homeostasis in the steady state $(9,10)$. IDCs recognize 'danger'-associated signals including pathogen-associated molecular patterns (PAMPs) and damage-associated molecular patterns (DAMPs) through pattern recognition receptors (PPRs), such as Toll-like receptors (TLRs) and NOD-like receptors. IDCs response to these signals and change to mDCs (11). MDCs show the high status of activation and capable of promoting $\mathrm{T}$ cell polarization toward to type $1 \mathrm{~T}$ helper (Th1), Th2, Th17 or regulatory $\mathrm{T}$ (Treg) cells $(7,12)$. Therefore, dynamic regulation of DCs maturation is essential for controlling immune-induced diseases.

The high-throughput platforms for analysis of gene expression, such as gene microarray technology and high-throughput sequence, have been used for more than ten years. These high-throughput analyses are broadly used as effective methods for obtaining the pathology-associated changes in the transcriptome level. A large number of gene expression profiling studies have been performed and covered thousands of differentially expressed genes (DEGs) in pathways, biological process or molecular functions. However, comparative analysis of DEGs is limited because of tissue or sample heterogeneity in independent studies. Therefore, the integrated bioinformatics methods combining with different expression profiling are used to identify hub genes in many diseases. The integrated bioinformatics methods are widely performed in cancer. Guo et al (13), found that potential candidate biomarkers for diagnosis, drug targets and prognosis in colorectal cancer. Dong et al (14), analyzed two expression profiles and explored five hub genes as critical biomarkers of osteosarcoma. However, the interactions among DEGs and the pathways in DCs remain unclear.

In this investigation, we assayed four microarray datasets including GSE52894, GSE72893, GSE75938 and GSE77969 from Gene Expression Omnibus (GEO, https://www.ncbi.nlm.nih.gov/geo/), from which there are total of $14 \mathrm{mDCs}$ samples and $14 \mathrm{imDCs}$ samples available. Gene expression profiles of mDCs were compared with imDCs to identify the DEGs. Subsequently, the DEGs were screened using DAVID Bioinformatics Resources 6.8 for Gene Ontology (GO) and pathway enrichment analysis. Furthermore, we identified ten hub genes (ISG20, IFITM1, HLA-F, IRF1, USP18, IFI44L, GBPI, IFI35, IFI27, IFI6) through protein-protein interaction (PPI) and modular analysis. Our results suggested that data mining and integration could be a useful method to understand the mechanism and development of DCs in immune-induced diseases.

\section{Materials and methods}

Microarray data information. The gene expression profiles of GSE52894, GSE72893, GSE75938 and GSE77969 were downloaded from GEO (https://www.ncbi.nlm.nih.gov/geo/geo2r/). The gene expression profiles GSE52894 were measured with GPL10558 platforms (Illumina HumanHT-12 v4.0 expression beadchip) and included sixteen samples from four monDCs types (immature, mature, tolerogenic and LPS-tolerogenic). The microarray data of GSE72893 was based on GPL10558 platforms (Illumina HumanHT-12 v4.0 expression beadchip) and consisted of four imDCs samples, four mature DCs samples and two Treg-conditioned DCs samples. GSE75938 was based on GPL15207 platforms (Affymetrix Human Gene Expression Array). The GSE75938 dataset contained 14 samples, including three sets of monocytes, derived imDCs and macrophage, mature DCs and macrophage. GSE77969 which was based on GPL13667 platforms (Affymetrix Human Genome U219 Array) consisted of three imDCs samples and three mature DCs samples. We chose imDCs and mature DCs samples from these 4 datasets for integrated analysis in the present study.

Identification of DEGs. GEO2R (www.ncbi.nlm.nih. gov/geo/geo2r/) is an interactive web tool in order to identify genes that are differentially expressed across experimental conditions using the GEOquery and limma R packages from the Bioconductor project. We screened DEGs between imDCs and mature DCs in these four datasets by GEO2R. The P-value was adjusted for the correction of false positive results when using the Benjamini and Hochberg false discovery rate method by default. According to other studies, we used log transformation to identify DEGs with adjusted P-value $<0.01$ and $\mid \log \mathrm{FCl}>1$.

Gene ontology and pathway enrichment analysis. The GO and Kyoto Encyclopedia of Genes and Genomes (KEGG) of candidate DEGs were analyzed by DAVID Bioinformatics Resources 6.8 (david.ncifcrf.gov//tools.jsp). DAVID is a website for high-throughput functional annotation analysis. A P-value $<0.05$ was set as the cut-off criterion.

PPI network and module selection. We used search tool for the retrieval of interacting genes 10.5 (STRING, https://string-db.org/) to evaluate the interactive relationships among DEGs. The combined score $>0.4$ was set as the cut-off criterion. PPI networks were constructed and analyzed using Cytoscape v3.5.1 software. The plug-in molecular complex detection (MCODE) was performed to screen modules of PPI network in Cytoscape. The criteria were set as follows: degree cutoff $=2$, node score cutoff $=0.2, \mathrm{k}$-core $=2$, max. depth $=100$. MCODE scores $>3$ and number of nodes $>4$.

Generation of human monocyte-derived DCs. Human peripheral blood was isolated from healthy volunteers by buffy coats and DCs were generated as previously described (15). Briefly, peripheral blood mononuclear cells (PBMCs) were isolated using Ficoll-Hypaque centrifugation (TBDscience, China) according to the manufacturer's instructions. The project was approved by the Clinical Research Ethics Committee of the Second Affiliated Hospital of Harbin Medical University and written informed consent was obtained from all volunteers. PBMCs were seeded at a concentration of $6 \times 10^{6}$ cells per well in RMPI 1640 (Hyclone; GE Healthcare, Chicago, IL, USA) media with 10\% fetal bovine serum (ScienCell Research Laboratories, Inc., San Diego, CA, USA), penicillin (100 U/ml; Beyotime Institute of Biotechnology, Haimen, China) and streptomycin (100 ug/ml; Beyotime Institute of Biotechnology) for $2 \mathrm{~h}$ at $37^{\circ} \mathrm{C}$. Then, non-adherent cells were removed by washing. The adherent cells were cultured with human recombinant IL-4 $(50 \mathrm{ng} / \mathrm{ml}$; PeproTech, Inc., Rocky Hill, NJ, USA) and GM-CSF (100 ng/ml; 
Table I. List of primers used for reverse transcription-quantitative polymerase chain reaction.

\begin{tabular}{lll}
\hline Name & \multicolumn{1}{c}{ Sense sequence $\left(5^{\prime}-3 '\right)$} & \multicolumn{1}{c}{ Antisense sequence $\left(5^{\prime}-3^{\prime}\right)$} \\
\hline ISG20 & GCTTGCCTTTCAGGAGCTG & ATCACCGATTACAGAACCCG \\
IFITM1 & CCTCTTCTTGAACTGGTGCTGTCTG & CGTCGCCAACCATCTTCCTGTC \\
HLA-F & TGATCTCCGCAGGGTAGAAG & AATGGGAAGGAGACGCTACA \\
IRF1 & ATCCTTGTTGATGTCCCAGC & GACCCTGGCTAGAGATGCAG \\
USP18 & TCAGGACAGCACGACTTCAC & CGGAACTTCGGTCCCAG \\
IFI4L & TTCCATGTCAATCTTGTTGTCAC & TTTCTGTCTCCAAACCGTGG \\
GBP1 & GCAGAACTAGGATGTGGCCT & AACAAGCTGGCTGGAAAGAA \\
IFI35 & CCCACAGCCTCATCTTGAGT & TCTGAAGCCTCAGCTCTTGC \\
IFI27 & CCACAACTCCTCCAATCACA & GCCTCTGCTCTCACCTCATC \\
IFI6 & GTGGCAGCAGCGTCGTCATAG & GGCTACTCCTCATCCTCCTCACTATC \\
ACTB & CGTGGACATCCGCAAAGA & GAAGGTGGACAGCGAGGC
\end{tabular}

ISG20, interferon-stimulated gene of $20 \mathrm{kDa}$ protein; IFITM1, interferon induced transmembrane protein 1; HLA-F, human leukocyte antigen F; IRF1, interferon regulatory factor 1; USP18, ubiquitin-specific peptidase 18; IFI44L, interferon-induced protein 44-like; GBP1, Guanylate-binding protein 1; IFI35, interferon-induced protein 35; IFI27, interferon-a-inducible protein 27; IFI6, interferon- $\alpha$-inducible protein 6 ; ACTB, $\beta$-actin.

PeproTech, Inc.) in order to obtain immature monocyte-derived dendritic cells (Mo-DCs). The half of medium was changed every other day. Cells were stimulated with lipopolysaccharides (LPS) (200 ng/ml; Sigma-Aldrich; Merck KGaA, Darmstadt, Germany) on 6 days for $24 \mathrm{~h}$ for inducing maturation DCs.

Reverse transcription-quantitative transcriptase polymerase chain reaction ( $R T-q P C R)$. Total RNA was extracted from cells using TRIzol reagents (Invitrogen; Thermo Fisher Scientific, Inc., Waltham, MA, USA) and the firststrand complementary DNAs (cDNAs) were synthesized with a Transcriptor First Strand cDNA Synthesis kit (Roche Diagnostics, Basel, Switzerland) according to the manufacturer's instructions. The RT reactions were carried out for $60 \mathrm{~min}$ at $50^{\circ} \mathrm{C}$ and $5 \mathrm{~min}$ at $85^{\circ} \mathrm{C}$. The PCR protocol consisted of 40 cycles of $10 \mathrm{sec}$ at $95^{\circ} \mathrm{C}, 30 \mathrm{sec}$ at $60^{\circ} \mathrm{C}$ and $30 \mathrm{sec}$ at $72^{\circ} \mathrm{C}$ using Bestar SybrGreen qPCR Master Mix (DBI Bioscience, Germany). All reactions were measured in triplicate. The primers used for RT-qPCR were in Table I. The expression of hug genes relative to $A C T H$ were analyzed using the $2-\Delta \Delta C q$ method. All the data were given in terms of relative mRNA expression level as means $\pm \mathrm{SD}$ (standard deviation). $\mathrm{P}<0.05$ was considered to indicate a statistically significant difference.

MicroRNA regulatory analysis. MiRTarBase is the experimentally validated microRNA-target interactions database. We used miRTarBase release 6.0 to identify target genes among DEGs specifically for important microRNAs in DCs. The microRNA-targets regulatory network was constructed for DCs by Cytoscape.

Statistical analysis. GraphPad Prism 6 (GraphPad Software, Inc., La Jolla, CA, USA) was used to analyze the data. All data are expressed as the mean \pm standard deviation. For three independent experiments, a two-tailed Student's t-test was used to evaluate the differences between imDC and mDC. $\mathrm{P}<0.05$ was considered to indicate a statistically significant difference

\section{Results}

Identification of DEGs in $m D C s$. The raw data file of GES52894, GSE72893, GSE75938 and GSE77969 were uploaded to GEO2R to screen DEGs between immature and mature DCs. 14 imDCs samples and $14 \mathrm{mDCs}$ samples were analyzed. A total of 1057, 642, 699 and 1661 DEGs were up-regulated in the GES52894, GSE72893, GSE75938 and GSE77969 datasets (Fig. 1A). In addition, 1088, 716, 1759 and 2784 DEGs were down-regulated in GES52894, GSE72893, GSE75938 and GSE77969 datasets, respectively (Fig. 1B). After integrated bioinformatical analysis, 596 DEGs were identified from the four profile datasets (data not shown), including 241 upregulated genes and 365 downregulated genes in the mDCs compared to imDCs (Fig. 1).

DEGs gene ontology enrichment analysis. To explore the possible functional annotation and pathway enrichment of DEGs, we respectively uploaded the upregulated genes and downregulated genes to online software DAVID. The top five terms enriched were selected in each category according to P-value. GO biological processes (BP) analysis showed that the upregulated DEGs were significantly enriched in defense response to virus, apoptotic process, type I interferon signaling pathway, negative regulation of viral genome replication and inflammatory response (Fig. 2), while the downregulated DEGs were enriched in oxidation-reduction process, antigen processing, ATP synthesis coupled proton transport, regulation of release of sequestered calcium ion into cytosol and NAD metabolic process (Fig. 3). For cell component (CC), the upregulated DEGs were enriched in cytosol, cytoplasm, I-kappaB/NF-kappaB complex, host cell and external side of plasma membrane (Fig. 2); The downregulated DEGs were enriched in extracellular exosome, integral component of membrane, endosome membrane, cytosol and membrane (Fig. 3). In addition, the molecular function (MF) of upregulated DEGs were mainly associated with TAP1 binding, protein 

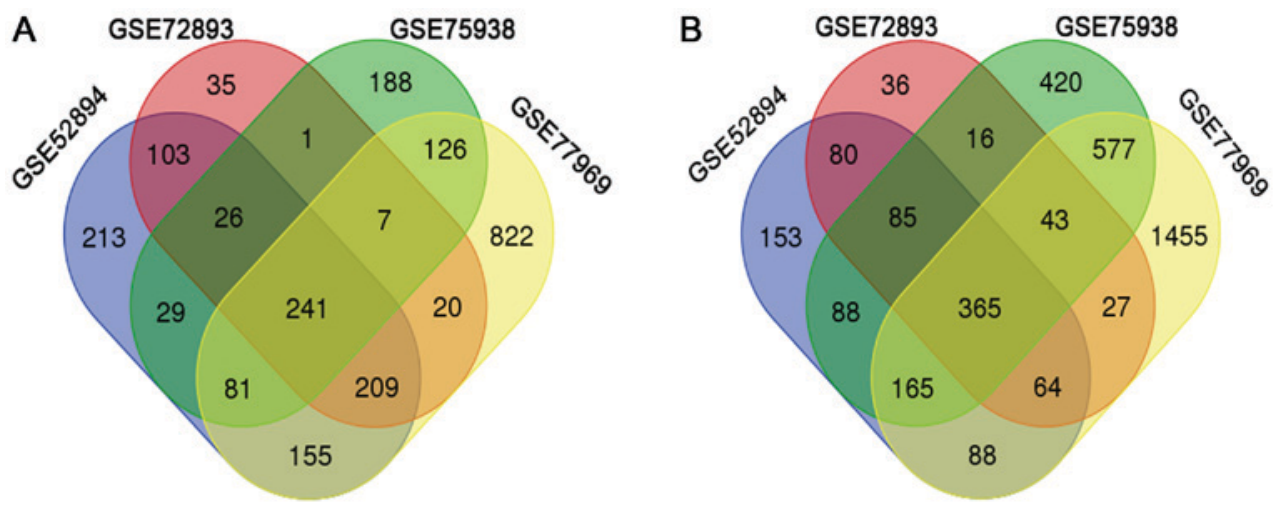

Figure 1. Venn diagram analysis of DEGs from the GSE52894, GSE72893, GSE75938 and GSE77969 datasets. (A) A total of 241 upregulated genes were identified in all 4 datasets. (B) A total of 365 genes were downregulated in all 4 datasets. The different colors represent the different datasets. The DEGs were identified with an adjusted $\mathrm{P}$-value of $<0.01$ and $\mid \log \mathrm{FCl}>1$. DEGs, differentially expressed genes.

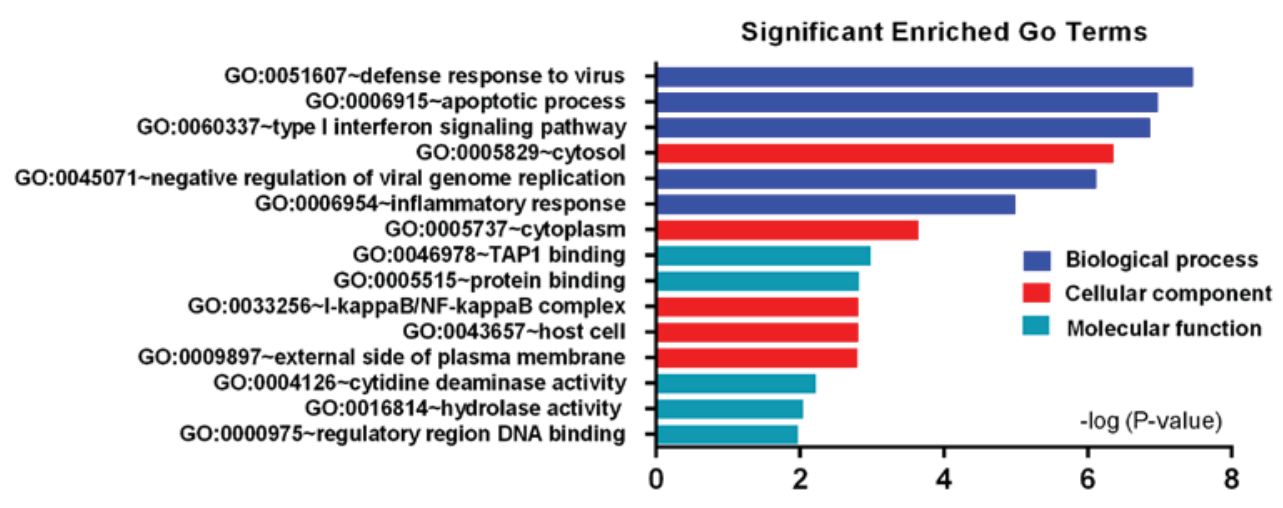

Figure 2. GO enrichment analysis of upregulated DEGs. GO analysis classified the DEGs into 3 groups, including biological process, cellular component and molecular function. GO, Gene Ontology; DEGs, differentially expressed genes.

binding, cytidine deaminase activity, hydrolase activity and regulatory region DNA binding (Fig. 2), while the downregulated DEGs were involved in beta-2-microglobulin binding, endogenous and exogenous lipid antigen binding, lipopeptide binding and protein binding (Fig. 3). These results showed that the DEGs were involved in the regulation of immune response.

KEGG pathway analysis. The most significantly enriched pathways of the upregulated and downregulated DEGs were shown in Fig. 4. The upregulated DEGs mainly enriched in TNF signaling pathway, NF-kappa B signaling pathway, RIG-I-like receptor signaling pathway, NOD-like receptor signaling pathway and viral carcinogenesis (Fig. 4A). The down-regulated DEGs were enriched in lysosome, transcriptional misregulation in cancer, hematopoietic cell lineage, HTLV-I infection and valine, leucine and isoleucine degradation (Fig. 4B). These enrichment analysis suggested that the regulation and function of DEGs was mainly in response to inflammatory.

PPI network construction and modules selection. To understand cellular functions and biological processes, we constructed the PPI network of DEGs using STRING and Cytoscape software. The PPI network consisted of 606 nodes and 1632 edges (data not shown). The top 10 hub genes with higher degrees were screened including ISG20, IFITMI,
HLA-F, IRF1, USP18, IFI44L, GBP1, IFI35, IFI27, IFI6. Then, we verified the expression of the top 10 hub genes in mDCs by RT-qPCR. These 10 hub genes were upregulated in mDCs compared with imDCs (Fig. 5A-J). Moreover, the top 2 significant modules were selected from the DEGs PPI network using plug-ins MCODE (Fig. 6A and B). Module 1 included 15 nodes and 93 edges and module 2 had 28 nodes and 101 edges. Furthermore, functional and pathway enrichment analysis of genes in these two modules were performed using DAVID. The results showed that Module 1 were mainly associated with type I interferon signaling pathway, defense response to virus, regulatory region DNA binding and RIG-I-like receptor signaling pathway, while genes in Module 2 were mainly enriched in inflammatory response, apoptotic process, immune response, NF-kappa B signaling pathway and TNF signaling pathway (Table II). These results indicate that the hub genes which we screened from the PPI network play an important role in the immune response of DCs.

MicroRNA regulatory network analysis. MicroRNAs (miRNAs) inhibit gene expressing by binding to complementary 3'-untranslated regions of mRNA and degrading specific target mRNA. A growing body of evidence has demonstrated that miRNAs play an important role in the immune response of DCs (16). In a previous study, we demonstrated that let-7i-5p depress maturation of DCs via targeting SOCS1 (7). 


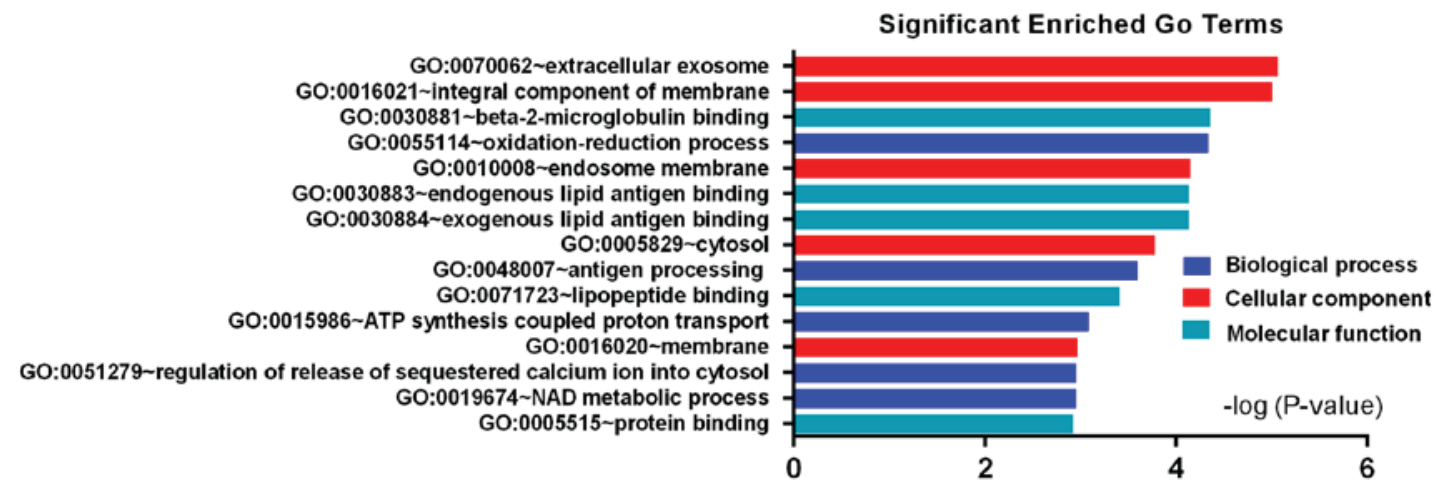

Figure 3. GO enrichment analysis of downregulated DEGs. GO analysis classified the DEGs into 3 groups, including biological process, cellular component and molecular function. GO, Gene Ontology; DEGs, differentially expressed genes.

A

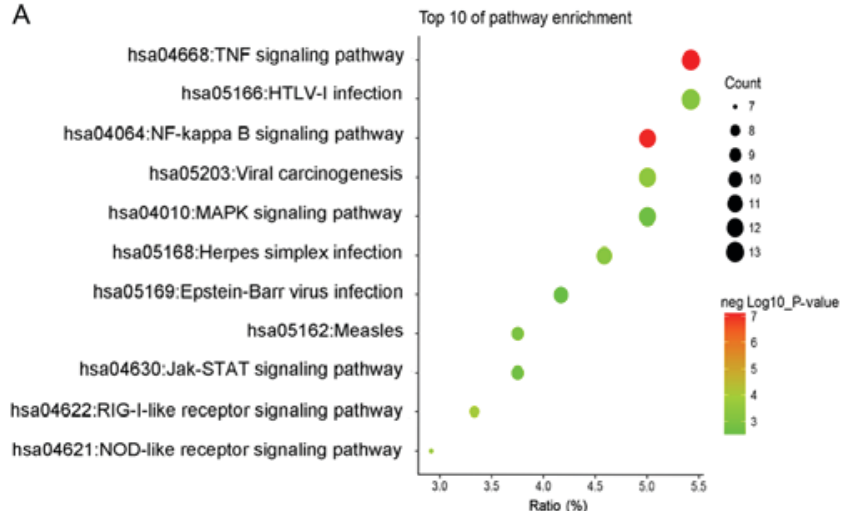

B

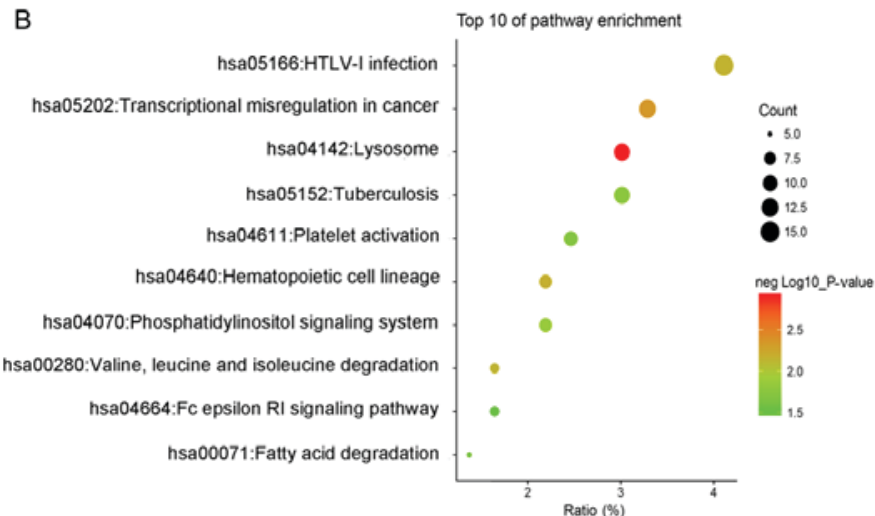

Figure 4. Kyoto Encyclopedia of Genes and Genomes pathways analysis of (A) upregulated and (B) downregulated genes in mDCs. The top 10 terms were selected according to P-value. mDCs, mature dendritic cells.

$M i R-155-5 p$ is a key negative regulator controlling IL-1 $\beta$ and other inflammatory cytokines produced during activation of DCs (17). Several studies have demonstrated that miR-34a-5p impact on the maturation of DCs $(18,19)$. Therefore, we selected let-7i-5p, miR-155-5p and miR-34a-5p for further analysis. We identified miRNAs-mRNA network from the downregulated DEGs because miRNAs suppress the target gene expression. The miRNAs-DEGs regulatory network of mDCs included 63 pairs of regulatory relationship combined with 3 miRNAs and 58 regulatory genes (Fig. 7). Through the construction of miRNAs-DEGs network, we screened the DEGs can provide new ideas for the treatment of immune response in DCs.

\section{Discussion}

Many diseases, such as atherosclerosis, GVHD, cancer, rheumatic diseases, are caused by abnormal function of the immune system. DCs play a central role in immune-induced diseases, the dynamic control state of DCs maturation may be a key factor for these diseases. Therefore, understanding the regulation mechanism of DCs maturation is essential to intervene the progress of diseases. Numerous studies have been conducted to reveal the mechanism of DCs maturation, but most studies focus on a single genetic event. In the present study, we screened four gene expression profiles (GSE52894, GSE72893, GSE75938 and GSE77969) and deeply analyzed these datasets using bioinformatics methods. A total of 596
DEGs were identified, consisting of 241 upregulated genes and 365 downregulated genes in mDCs. Function annotation and KEGG pathway enrichment analysis showed that the upregulated DEGs were mainly enriched in apoptotic process, type I interferon signaling pathway, inflammatory response, TNF signaling pathway and NF-kappa B signaling pathway; while the downregulated DEGs were mainly involved in oxidation-reduction process, antigen processing, regulation of release of sequestered calcium ion into cytosol, lysosome and HTLV-I infection. This is consistent with the knowledge that the NF-kappa B signaling pathway, TNF signaling pathway and type I interferon signaling pathway are the main pathway for mDCs (20-24). These results indicated that the DEGs were mainly focused on the immune response. Therefore, monitoring these signaling pathways may suppress the maturation of DCs.

Based on the PPI network, we finally identified 10 hub genes: ISG20, IFITM1, HLA-F, IRF1, USP18, IFI44 L, GBP1, IFI35, IFI27 and IFI6. They were maybe the therapeutic molecular targets for immune-induced diseases. ISG20 was interferon stimulated exonuclease gene 20 which is involved in defense response to virus and type I interferon signaling pathway. DCs play a crucial role in presenting viral antigens and inducing adaptive immune responses that eliminate the virus (25). Most studies have demonstrated that ISG20 can suppress virus replication. Leong et al (26), reported that ISG20 can selectively degrade HBV RNA and blocks replication of infectious HBV 

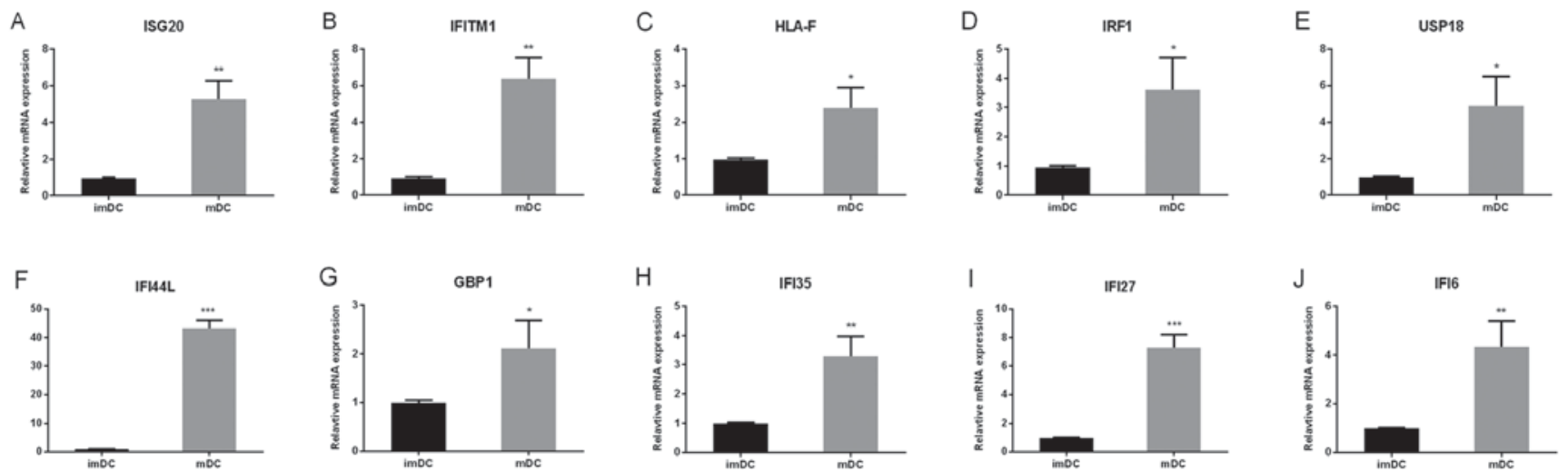

Figure 5. Top 10 hub genes were detected by reverse transcription-quantitative polymerase chain reaction. The expression of the top 10 hub genes, (A) ISG20, (B) IFITM1, (C) HLA-F, (D) IRF1, (E) USP18, (F) IFI44L, (G) GBP1, (H) IFI35, (I) IFI27 and (J) IFI6 were upregulated in mDCs. The data are presented as the mean \pm standard deviation of 3 independent experiments. ${ }^{*} \mathrm{P}<0.05,{ }^{* * *} \mathrm{P}<0.01$ and ${ }^{* * * *} \mathrm{P}<0.001$, vs. the imDCs group. DCs, dendritic cells; $\mathrm{mDCs}$, mature $\mathrm{DCs}$; imDCs, immature DCs; ISG20, interferon-stimulated gene of $20 \mathrm{kDa}$ protein; IFITM1, interferon induced transmembrane protein 1; HLA-F, human leukocyte antigen F; IRF1, interferon regulatory factor 1; USP18, ubiquitin-specific peptidase 18; IFI44L, interferon-induced protein 44-like; GBP1, Guanylate-binding protein 1; IFI35, interferon-induced protein 35; IFI27, interferon-a-inducible protein 27; IFI6, interferon- $\alpha$-inducible protein 6.
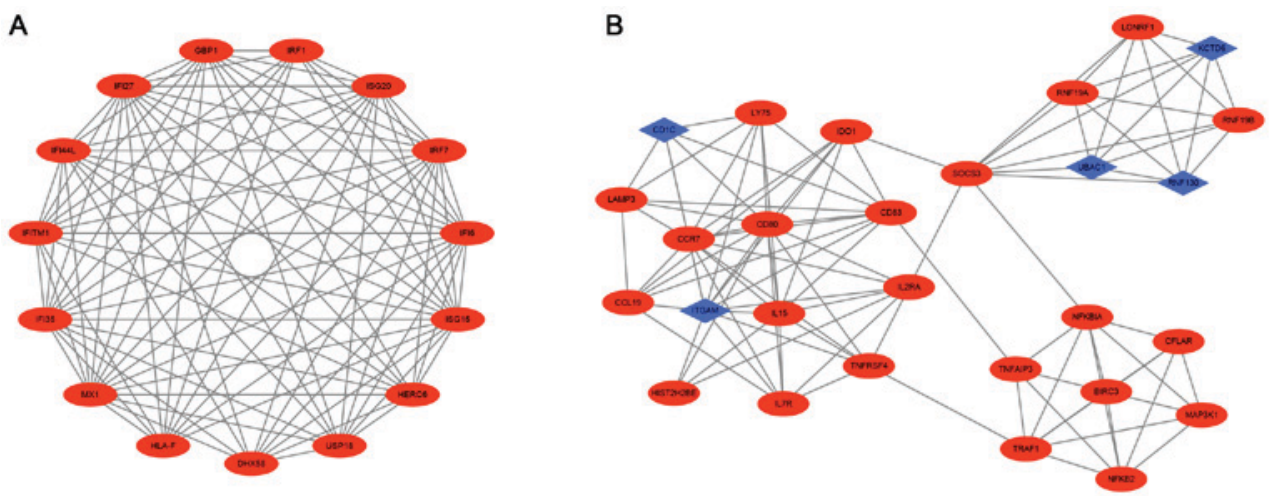

Figure 6. Top 2 modules from the protein-protein network according to the Molecular Complex Detection score. (A) Module 1 and (B) Module 2. Red indicates upregulated genes and the blue indicates downregulated genes.

particles. It has also demonstrated that ISG20 may represent a maker in chronic hepatitis B patients, which was associated with a favorable response to IFN- $\alpha$ therapy (27). Moreover, Zahoor et al (28) reported that $I S G 20$ was upregulated in $\mathrm{mDCs}$ treated with HIV-1 Vpr. Thus, we supposed that the ISG20 may play an important role in immune-induced diseases. The second hub gene interferon induced transmembrane protein 1 (IFITMI) is a member of the IFN-inducible transmembrane protein family. IFITM1 controls proliferation, homotypic adhesion in lymphocytes and metastasis $(29,30)$. Overexpression of IFITMI can negatively regulate cell growth (31). A wide range of viruses can be inhibited by IFITM1 through immune responses, such as hepatitis C virus, hepatitis B virus, H5N1 virus and HIV (32). Zhang et al (33), reported that IFITMI was significantly increased in human mo-DCs treated with dengue virus. It has also reported that IFITMI was one of the up-regulated interferon-inducible antiviral proteins in LPS-stimulation DCs (34). These results suggested that IFITMI in this study induced maturation of DCs. Human leukocyte antigen F $(H L A-F)$, a non-classical MHC molecule, is expressed on proliferating lymphoid and monocyte cells as a protective molecule in a novel pathway for Ag cross-presentation $(35,36)$. Goodridge et al (35), showed that $H L A-F$ may act in an immunoregulatory capacity centered on inflammatory response. $H L A-F$ were up-regulated in many immune-induced diseases, such as coronary heart disease (37), SLE (38). Intriguingly, our study for the first time has documented that $H L A-F$ was up-regulated during maturation of DCs. However, the precise function of HLA-F in DCs remains elusive, we need to completely decipher the mechanism of $H L A-F$ in DCs. Interferon regulatory factor $1(I R F-1)$, a kind of transcription factors, is the first discoverable member of the interferon regulatory factor family (39). The level of IRF-I was regulated by various stimuli including IFN (type I and II), cytokines, double-stranded RNA. IRF-1 plays an important role in many physiological and pathological aspects, such as proinflammatory injury, development of immune system, autoimmunity and viral infection (39). The tick-borne encephalitis virus, a leading cause of viral encephalitis, can inhibit DCs maturation by diminished the protein of IRF-1 and nuclear localization (23). NF- $\kappa \mathrm{B}$ activated by TNF- $\alpha$ could increase the expression of $I R F-1$ and induced the maturation of DCs marker CD25, CD40 (40). Interestingly, IRF-1 can regulate the expression of other hub genes, ISG20, USPI8 and GBPI (41-43). Thus, IRF-1 may exert an enormous function on regulating maturation of DCs. Ubiquitin-specific peptidase 18 (USP18), a member of USP family, is a negative regulator of type I and type 
Table II. Functional and pathway enrichment analysis of the genes in modules.

\begin{tabular}{|c|c|c|c|c|}
\hline Category & Term & Gene function & $\begin{array}{l}\text { Gene } \\
\text { count }\end{array}$ & P-value \\
\hline \multicolumn{5}{|l|}{ A, Module 1} \\
\hline GOTERM_BP_DIRECT & GO:0060337 & Type I interferon signaling pathway & 10 & $1.86 \times 10^{19}$ \\
\hline GOTERM_BP_DIRECT & GO:0051607 & Defense response to virus & 8 & $2.52 \times 10^{11}$ \\
\hline GOTERM_BP_DIRECT & GO:0009615 & Response to virus & 5 & $1.66 \times 10^{6}$ \\
\hline GOTERM_BP_DIRECT & GO:0045071 & Negative regulation of viral genome replication & 4 & $4.48 \times 10^{6}$ \\
\hline GOTERM_BP_DIRECT & GO:0060333 & Interferon- $\gamma$-mediated signaling pathway & 4 & $2.55 \times 10^{5}$ \\
\hline GOTERM_CC_DIRECT & GO:0005829 & Cytosol & 8 & 0.006784 \\
\hline GOTERM_MF_DIRECT & GO:0000975 & Regulatory region DNA binding & 2 & 0.00991 \\
\hline GOTERM_MF_DIRECT & GO:0005525 & GTP binding & 3 & 0.03921 \\
\hline KEGG_PATHWAY & hsa04622 & RIG-I-like receptor signaling pathway & 3 & $9.92 \times 10^{4}$ \\
\hline
\end{tabular}

B, Module 2

\begin{tabular}{|c|c|c|c|c|}
\hline GOTERM_BP_DIRECT & GO:0006954 & Inflammatory response & 8 & $1.70 \times 10^{6}$ \\
\hline GOTERM_BP_DIRECT & GO:0006915 & Apoptotic process & 8 & $2.38 \times 10^{5}$ \\
\hline GOTERM_BP_DIRECT & GO:0006955 & Immune response & 7 & $4.54 \times 10^{5}$ \\
\hline GOTERM_BP_DIRECT & GO:0032735 & Positive regulation of interleukin-12 production & 3 & $7.30 \times 10^{4}$ \\
\hline GOTERM_BP_DIRECT & GO:0010803 & $\begin{array}{l}\text { Regulation of tumor necrosis factor-mediated } \\
\text { signaling pathway }\end{array}$ & 3 & 0.001053 \\
\hline GOTERM_CC_DIRECT & GO:0009897 & External side of plasma membrane & 5 & $2.22 \times 10^{4}$ \\
\hline GOTERM_CC_DIRECT & GO:0005829 & Cytosol & 12 & 0.003709 \\
\hline GOTERM_CC_DIRECT & GO:0009986 & Cell surface & 5 & 0.006877 \\
\hline GOTERM_CC_DIRECT & GO:0005737 & Cytoplasm & 14 & 0.017334 \\
\hline GOTERM_MF_DIRECT & GO:0004842 & Ubiquitin-protein transferase activity & 6 & $1.05 \times 10^{4}$ \\
\hline GOTERM_MF_DIRECT & GO:0016874 & Ligase activity & 6 & $6.21 \times 10^{4}$ \\
\hline GOTERM_MF_DIRECT & GO:0008270 & Zinc ion binding & 8 & 0.001191 \\
\hline GOTERM_MF_DIRECT & GO:0031624 & Ubiquitin conjugating enzyme binding & 2 & 0.044943 \\
\hline KEGG_PATHWAY & hsa04064 & $\mathrm{NF}-\kappa \mathrm{B}$ signaling pathway & 7 & $7.96 \times 10^{8}$ \\
\hline KEGG_PATHWAY & hsa04668 & TNF signaling pathway & 7 & $2.61 \times 10^{7}$ \\
\hline KEGG_PATHWAY & hsa04060 & Cytokine-cytokine receptor interaction & 6 & $3.10 \times 10^{4}$ \\
\hline KEGG_PATHWAY & hsa04640 & Hematopoietic cell lineage & 4 & 0.00151 \\
\hline KEGG_PATHWAY & hsa05166 & HTLV-I infection & 5 & 0.004594 \\
\hline
\end{tabular}

If there were $>5$ terms enriched in this category, the top 5 terms were selected according to P-values. Gene count values represent the number of enriched genes in each term. GO, Gene Ontology; KEGG, Kyoto Encyclopedia of Genes and Genomes; RIG-I, retinoic-acid-inducible protein 1; NF, nuclear factor; TNF, tumor necrosis factor; HTLV-I, human T-cell leukemia-lymphoma virus type 1.

III interferon signaling (44). USP18 regulates various immunological processes, including autoimmune diseases, pathogen control and cancer development. Cong et al (45), demonstrated that USP18 can promote DCs development. USP18-/- mice bone marrow-derived DCs was reduced because of high expression of GM-CSF signaling inhibitors SOCS1/SOCS3 (45). In addition, lack of USP18 reduced the number of DCs and enhanced the expression of MHC I and the costimulatory molecular CD80 (46). Guanylate-binding protein 1 (GBPl) was associated with the control of immune innate response to foreign antigens (47). Thomas has demonstrated that GBPl DNA was isolated from a human genomic library and mapped to human chromosome 1 (48). Kim et al (47), showed that GBPI contributed to vascular dysfunction in chronic inflammatory diseases by inhibiting the proliferation and migration of endothelial progenitor cells. GBP1 was up-regulated in stimulated T-cell treated with phytohaemagglutinin (49). GBP1, a classical mature DCs biomarker, was up-regulated throughout maturation of DCs (50). The mechanism of USP18 and GBPI in maturation DCs remains unknown.

In the present study, we found that four interferon inducible proteins, IFI44L, IFI35, IFI27 and IFI6, were up-regulated in mDCs. IFI44L, IFI35, IFI27 and IFI6 were key regulatory targets in immune-induced diseases, such as SLE, rheumatoid arthritis and myelofibrosis (51-53). Interestingly, IFI44L was the highest up-regulated genes 


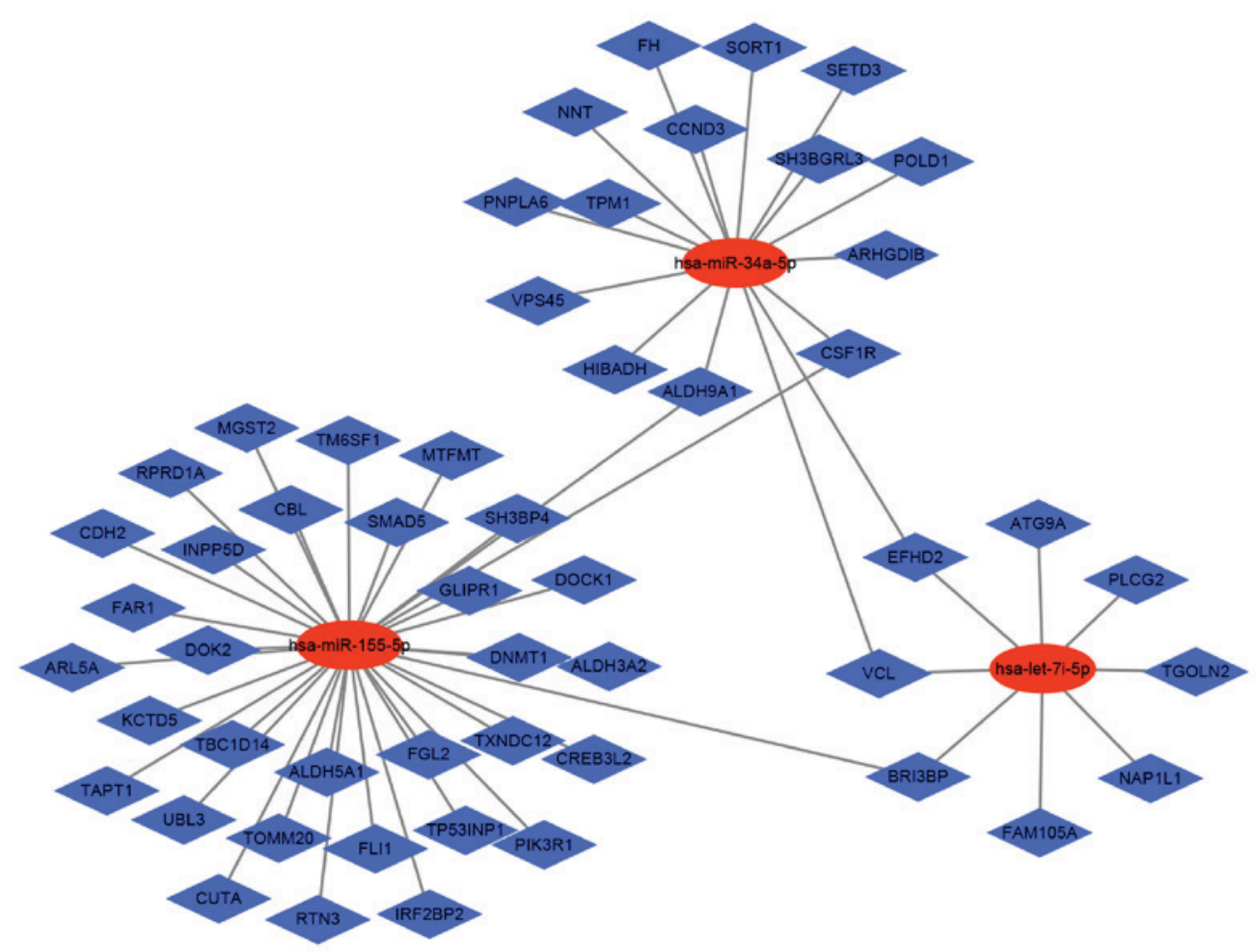

Figure 7. miRNAs-mRNAs regulatory network analysis. The miRNAs presented in the figure. are associated with the maturation of dendritic cells. Red indicates miRNAs and blue represents the downregulated differentially expressed genes. miRNA, microRNA.

among the ten hub genes validated by RT-qPCR. Aida demonstrated that IFI44L and IFI27 were up-regulated in human DCs treated with HIV-1 Vpr (28). In other studies, IFI27 was up-regulated in DCs with different stimuli $(54,55)$. However, the biological function of IFI35 and IFI6 in DCs were unclear. Thus, further researches are needed to understand the mechanism of four interferon inducible proteins in DCs. Taken together, these data suggested that these ten hub genes were up-regulated and highly connected. The hub genes may be involved in the regulation of maturation of DCs via the checkpoint mechanism.

Module analysis of the PPI network revealed that module 1 contained the ten hub genes, suggesting that these ten hub genes had close interaction and together determined the key pathway during the maturation of DCs. Functional and pathway enrichment analysis of genes in module 1 and 2 was mainly involved in type I interferon signaling, NF-kappa B signaling pathway, TNF signaling pathway. Studies have demonstrated that type I interferon signaling, NF-kappa B signaling pathway, TNF signaling pathway play an important role in mDCs (56-58). Thus, we propose that these molecular pathways in mDCs might represent the promising candidates for pharmacologic evaluation and for therapeutic intervention in immune-induced diseases.

In conclusion, we have identified 596 DEGs, 606 nodes, 1632 edges and 10 hub genes by multiple cohorts profile datasets and integrated bioinformatical analysis in maturation DCs. The 10 hub genes significantly enriched in several pathways, including type I interferon signaling, NF-kappa B signaling pathway, TNF signaling pathway. Our study provided a set of candidate target genes for future investigation into the molecular mechanisms and biomarkers of mDCs.
These findings could significantly improve our understanding of DCs in immune-induced diseases.

\section{Acknowledgements}

The authors also would like to thank Dr. Yuanyuan Wei (China Pharmaceutical University, Jiangsu, China) for the excellent technical support and for critically reviewing the manuscript.

\section{Funding}

The present study was supported by The National Natural Science Foundation of China (grant nos. 81670373, 81330033, 81670459 and 81771946), The Natural Science Foundation of Heilongjiang Province of China (grant no. H2015048), and Key Laboratory of Myocardial Ischemia, Harbin Medical University, Ministry of Education, Heilongjiang Province, China (grant nos. KF201715, KF201716 and KF201717).

\section{Availability of data and materials}

The datasets used and/or analyzed during the current study are available from the corresponding author on reasonable request.

\section{Authors' contributions}

YZ and JW and BY conceived and designed the study; SL, ML, HZ and QY performed the experiments; and YZ, XZ, YS and $\mathrm{MZ}$ analyzed the data. $\mathrm{YZ}$ and $\mathrm{XZ}$ wrote the paper, and YS, JW and BY revised the manuscript and gave final approval of the version to be published. 


\section{Ethics approval and consent to participate}

The present study was approved by the Clinical Research Ethics Committee of the Second Affiliated Hospital of Harbin Medical University (Heilongjiang, China), and written informed consent was obtained from all participants.

\section{Consent for publication}

Written informed consent was obtained from all volunteers for the publication of any associated data.

\section{Competing interests}

The authors declare that they have no competing interests.

\section{References}

1. Steinman RM and Cohn ZA: Identification of a novel cell type in peripheral lymphoid organs of mice. I. Morphology, quantitation, tissue distribution. J Exp Med 137: 1142-1162, 1973.

2. Austyn JM: Dendritic cells in the immune system-history, lineages, tissues, tolerance and immunity. Microbiol Spectr 4, 2016 doi: 10.1128/microbiolspec.

3. Gautier EL, Huby T, Saint-Charles F, Ouzilleau B, Pirault J, Deswaerte V, Ginhoux F, Miller ER, Witztum JL, Chapman MJ and Lesnik P: Conventional dendritic cells at the crossroads between immunity and cholesterol homeostasis in atherosclerosis. Circulation 119: 2367-2375, 2009.

4. Stoneman V, Braganza D, Figg N, Mercer J, Lang R, Goddard M and Bennett M: Monocyte/macrophage suppression in CD11b diphtheria toxin receptor transgenic mice differentially affects atherogenesis and established plaques. Circ Res 100: 884-893, 2007.

5. Paulson KE, Zhu SN, Chen M, Nurmohamed S, Jongstra-Bilen J and Cybulsky MI: Resident intimal dendritic cells accumulate lipid and contribute to the initiation of atherosclerosis. Circ Res 106: 383-390, 2010.

6. Hansson GK and Hermansson A: The immune system in atherosclerosis. Nat Immunol 12: 204-212, 2011.

7. Zhang M, Liu F, Jia H, Zhang Q, Yin L, Liu W, Li H, Yu B and Wu J: Inhibition of microRNA let-7i depresses maturation and functional state of dendritic cells in response to lipopolysaccharide stimulation via targeting suppressor of cytokine signaling 1 . J Immunol 187: 1674-1683, 2011.

8. Sun Y, Jin X, Liu X, Liu X, Zhang M, Liu W, Li Z, Han N, Tan M, Chi D, et al: MicroRNA let-7i regulates dendritic cells maturation targeting interleukin-10 via the Janus kinase 1-signal transducer and activator of transcription 3 signal pathway subsequently induces prolonged cardiac allograft survival in rats. J Heart Lung Transplant 35: 378-388, 2016.

9. Audiger C, Rahman MJ, Yun TJ, Tarbell KV and Lesage S: The importance of dendritic cells in maintaining immune tolerance. J Immunol 198: 2223-2231, 2017.

10. Waisman A, Lukas D, Clausen BE and Yogev N: Dendritic cells as gatekeepers of tolerance. Semin Immunopathol 39: 153-163, 2017.

11. Motran CC, Ambrosio LF, Volpini X, Celias DP and Cervi L: Dendritic cells and parasites: From recognition and activation to immune response instruction. Semin Immunopathol 39: 199-213, 2017.

12. Malinova D, Fritzsche M, Nowosad CR, Armer H, Munro PM, Blundell MP, Charras G, Tolar P, Bouma G and Thrasher AJ: WASp-dependent actin cytoskeleton stability at the dendritic cell immunological synapse is required for extensive, functional T cell contacts. J Leukoc Biol 99: 699-710, 2016.

13. Guo Y, Bao Y, Ma M and Yang W: Identification of key candidate genes and pathways in colorectal cancer by integrated bioinformatical analysis. Int J Mol Sci 18: pii: E722, 2017.

14. Dong B, Wang G, Yao J, Yuan P, Kang W, Zhi L and He X Predicting novel genes and pathways associated with osteosarcoma by using bioinformatics analysis. Gene 628: 32-37, 2017.

15. Marzaioli V, Hurtado-Nedelec M, Pintard C, Tlili A, Marie JC, Monteiro RC, Gougerot-Pocidalo MA, Dang PM and El-Benna J: NOX5 and p22phox are 2 novel regulators of human monocytic differentiation into dendritic cells. Blood 130: 1734-1745, 2017.
16. Smyth L, Boardman D, Tung S, Lechler R and Lombardi G: MicroRNAs affect dendritic cell function and phenotype. Immunology 144: 197-205, 2015.

17. Ceppi M, Pereira P, Dunand-Sauthier I, Barras E, Reith W, Santos MA and Pierre P: MicroRNA-155 modulates the interleukin-1 signaling pathway in activated human monocyte-derived dendritic cells. Proc Natl Acad Sci USA 106: 2735-2740, 2009.

18. Huang A, Yang Y, Chen S, Xia F, Sun D, Fang D, Xiong S, Jin L and Zhang J: MiR-34a promotes DCs development and inhibits their function on T cell activation by targeting WNT1. Oncotarget 8: 17191-17201, 2017.

19. Kurowska-Stolarska M, Alivernini S, Melchor EG, Elmesmari A, Tolusso B, Tange C, Petricca L, Gilchrist DS, Di Sante G, Keijzer C, et al: MicroRNA-34a dependent regulation of AXL controls the activation of dendritic cells in inflammatory arthritis. Nat Commun 8: 15877, 2017.

20. Chae CS, Kim GC, Park ES, Lee CG, Verma R, Cho HL, Jun CD, Yoo YJ and Im SH: NFAT1 regulates systemic autoimmunity through the modulation of a dendritic cell property. J Immunol 199: 3051-3062, 2017.

21. Riol-Blanco L, Delgado-Martín C, Sánchez-Sánchez N, Alonso-C LM, Gutiérrez-López MD, Del Hoyo GM, Navarro J, Sánchez-Madrid F, Cabañas C, Sánchez-Mateos P and Rodríguez-Fernández JL: Immunological synapse formation inhibits, via NF-kappaB and FOXO1, the apoptosis of dendritic cells. Nat Immunol 10: 753-760, 2009.

22. Summers deLuca L and Gommerman J: Fine-tuning of dendritic cell biology by the TNF superfamily. Nat Rev Immunol 12: 339-351, 2012.

23. Robertson SJ, Lubick KJ, Freedman BA, Carmody AB and Best SM: Tick-borne flaviviruses antagonize both IRF-1 and type I IFN signaling to inhibit dendritic cell function. J Immunol 192: 2744-2755, 2014

24. Prete F, Catucci M, Labrada M, Gobessi S, Castiello MC, Bonomi E, Aiuti A, Vermi W, Cancrini C, Metin A, et al: Wiskott-Aldrich syndrome protein-mediated actin dynamics control type-I interferon production in plasmacytoid dendritic cells. J Exp Med 210: 355-374, 2013.

25. Neyt K and Lambrecht B: The role of lung dendritic cell subsets in immunity to respiratory viruses. Immunol Rev 255: 57-67, 2013.

26. Leong C, Funami K, Oshiumi H, Mengao D, Takaki H, Matsumoto M, Aly HH, Watashi K, Chayama K and Seya T: Interferon-stimulated gene of $20 \mathrm{kDa}$ protein (ISG20) degrades RNA of hepatitis B virus to impede the replication of HBV in vitro and in vivo. Oncotarget 7: 68179-68193, 2016.

27. Lu X, Qin B, Ma Q, Yang C, Gong XY and Chen LM: Differential expression of ISG20 in chronic hepatitis B patients and relation to interferon-alpha therapy response. J Med Virol 85: 1506-1512, 2013.

28. Zahoor M, Xue G, Sato H and Aida Y: Genome-wide transcriptional profiling reveals that HIV-1 Vpr differentially regulates interferon-stimulated genes in human monocyte-derived dendritic cells. Virus Res 208: 156-163, 2015.

29. Yu F, Xie D, Ng SS, Lum CT, Cai MY, Cheung WK, Kung HF, Lin G, Wang X and Lin MC: IFITM1 promotes the metastasis of human colorectal cancer via CAV-1. Cancer Lett 368: 135-143, 2015.

30. Narayana SK, Helbig KJ, McCartney EM, Eyre NS, Bull RA, Eltahla A, Lloyd AR and Beard MR: The interferon-induced transmembrane proteins, IFITM1, IFITM2 and IFITM3 inhibit hepatitis C virus entry. J Biol Chem 290: 25946-25959, 2015.

31. Yang $\mathrm{G}, \mathrm{Xu} \mathrm{Y}, \mathrm{Chen} \mathrm{X}$ and Hu G: IFITM1 plays an essential role in the antiproliferative action of interferon-gamma. Oncogene 26: 594-603, 2007.

32. Li K, Jia R, Li M, Zheng YM, Miao C, Yao Y, Ji HL, Geng Y, Qiao W, Albritton LM, et al: A sorting signal suppresses IFITM1 restriction of viral entry. J Biol Chem 290: 4248-4259, 2015.

33. Zhang J, Sze DM, Yung BY, Tang P, Chen WJ, Chan KH and Leung PH: Distinct expression of interferon-induced protein with tetratricopeptide repeats (IFIT) $1 / 2 / 3$ and other antiviral genes between subsets of dendritic cells induced by dengue virus 2 infection. Immunology 148: 363-376, 2016.

34. Ishii K, Kurita-Taniguchi M, Aoki M, Kimura T, Kashiwazaki Y, Matsumoto $\mathrm{M}$ and Seya T: Gene-inducing program of human dendritic cells in response to BCG cell-wall skeleton (CWS), which reflects adjuvancy required for tumor immunotherapy. Immunol Lett 98: 280-290, 2005.

35. Goodridge JP, Burian A, Lee N and Geraghty DE: HLA-F and MHC class I open conformers are ligands for NK cell Ig-like receptors. J Immunol 191: 3553-3562, 2013. 
36. Dulberger CL, McMurtrey CP, Hölzemer A, Neu KE, Liu V, Steinbach AM, Garcia-Beltran WF, Sulak M, Jabri B, Lynch VJ, et al: Human leukocyte antigen $\mathrm{f}$ presents peptides and regulates immunity through interactions with NK cell receptors. Immunity 46: 1018-1029.e7, 2017.

37. Zidi I, Kharrat N, Abdelhedi R, Hassine AB, Laaribi AB, Yahia HB, Abdelmoula NB, Abid L, Rebai A and Rizzo R: Nonclassical human leukocyte antigen (HLA-G, HLA-E and HLA-F) in coronary artery disease. Hum Immunol 77: 325-329, 2016.

38. Jucaud V, Ravindranath MH, Terasaki PI, Morales-Buenrostro LE, Hiepe F, Rose T and Biesen R: Serum antibodies to human leucocyte antigen (HLA)-E, HLA-F and HLA-G in patients with systemic lupus erythematosus (SLE) during disease flares: Clinical relevance of HLA-F autoantibodies. Clin Exp Immunol 183: 326-340, 2016.

39. Dou L, Liang HF, Geller DA, Chen YF and Chen XP: The regulation role of interferon regulatory factor-1 gene and clinical relevance. Hum Immunol 75: 1110-1114, 2014.

40. Hu Y, Park-Min K, Yarilina A and Ivashkiv L: Regulation of STAT pathways and IRF1 during human dendritic cell maturation by TNF-alpha and PGE2. J Leukoc Biol 84: 1353-1360, 2008.

41. Gongora C, Degols G, Espert L, Hua T and Mechti N: A unique ISRE, in the TATA-less human Isg20 promoter, confers IRF-1-mediated responsiveness to both interferon type I and type II. Nucleic Acids Res 28: 2333-2341, 2000.

42. Briken V, Ruffner H, Schultz U, Schwarz A, Reis LF, Strehlow I, Decker T and Staeheli P: Interferon regulatory factor 1 is required for mouse Gbp gene activation by gamma interferon. Mol Cell Biol 15: 975-982, 1995.

43. Potu H, Sgorbissa A and Brancolini C: Identification of USP18 as an important regulator of the susceptibility to IFN-alpha and drug-induced apoptosis. Cancer Res 70: 655-665, 2010.

44. Honke N, Shaabani N, Zhang DE, Hardt C and Lang KS: Multiple functions of USP18. Cell Death Dis 7: e2444, 2016.

45. Cong XL, Lo MC, Reuter BA, Yan M, Fan JB and Zhang DE: Usp18 promotes conventional CD11b+ dendritic cell development. J Immunol 188: 4776-4781, 2012.

46. Honke N, Shaabani N, Zhang DE, Iliakis G, Xu HC, Häussinger D, Recher M, Löhning M, Lang PA and Lang KS: Usp18 driven enforced viral replication in dendritic cells contributes to break of immunological tolerance in autoimmune diabetes. PLoS Pathog 9: e1003650, 2013.

47. Kim BH, Shenoy AR, Kumar P, Das R, Tiwari S and MacMicking JD: A family of IFN- $\gamma$-inducible $65-\mathrm{kD}$ GTPases protects against bacterial infection. Science 332: 717-721, 2011.

48. Strehlow I, Lohmann-Matthes ML and Decker T: The interferon-inducible GBP1 gene: Structure and mapping to human chromosome 1. Gene 144: 295-299, 1994.
49. Haudek-Prinz V, Klepeisz P, Slany A, Griss J, Meshcheryakova A, Paulitschke V, Mitulovic G, Stöckl J and Gerner C: Proteome signatures of inflammatory activated primary human peripheral blood mononuclear cells. J Proteomics 76: 150-162, 2012.

50. Jin P, Han T, Ren J, Saunders S, Wang E, Marincola FM and Stroncek DF: Molecular signatures of maturing dendritic cells: Implications for testing the quality of dendritic cell therapies. J Transl Med 8: 4, 2010.

51. Zhao M, Zhou Y,Zhu B, Wan M, Jiang T, Tan Q, Liu Y, Jiang J, Luo S, Tan Y, et al: IFI44L promoter methylation as a blood biomarker for systemic lupus erythematosus. Ann Rheum Dis 75: 1998-2006, 2016.

52. Weix J, Häupl T, Raio L, Villiger P and Förger F: The physiologic increase in expression of some type I IFN-inducible genes during pregnancy is not associated with improved disease activity in pregnant patients with rheumatoid arthritis. Transl Res 161: 505-512, 2013

53. Skov V, Larsen TS, Thomassen M, Riley CH, Jensen MK, Bjerrum OW, Kruse TA and Hasselbalch HC: Whole-blood transcriptional profiling of interferon-inducible genes identifies highly upregulated IFI27 in primary myelofibrosis. Eur J Haematol 87: 54-60, 2011.

54. Tang B, Shojaei M, Parnell G, Huang S, Nalos M, Teoh S, O'Connor K, Schibeci S, Phu AL, Kumar A, et al: A novel immune biomarker IFI27 discriminates between influenza and bacteria in patients with suspected respiratory infection. Eur Respir J 49: 1602098, 2017.

55. Xi Y, Troy N, Anderson D, Pena OM, Lynch JP, Phipps S, Bosco A and Upham JW: Critical role of plasmacytoid dendritic cells in regulating gene expression and innate immune responses to human rhinovirus-16. Front Immunol 8: 1351, 2017.

56. Veenhuis R, Freeman ZT, Korleski J, Cohen LK, Massaccesi G, Tomasi A, Boesch AW, Ackerman ME, Margolick JB, Blankson JN, et al: HIV-antibody complexes enhance production of type I interferon by plasmacytoid dendritic cells. J Clin Invest 127: 4352-4364, 2017.

57. Meng Y, Chen C, Liu Y, Tian C and Li H: Angiotensin II regulates dendritic cells through activation of NF-kB /p65, ERK1/2 and STAT1 pathways. Cell Physiol Biochem 42: 1550-1558, 2017.

58. Xu J, Eastman A, Flaczyk A, Neal LM, Zhao G, Carolan J, Malachowski AN, Stolberg VR, Yosri M, Chensue SW, et al: Disruption of early tumor necrosis factor alpha signaling prevents classical activation of dendritic cells in lung-associated lymph nodes and development of protective immunity against cryptococcal infection. Mol Biol 7: e00510, 2016. International (CC BY-NC-ND 4.0) License. 